\title{
A New Reliability Model Based on Lindley Distribution with Application to Failure Data
}

\author{
Norah N. Al-Mutairi ${ }^{D},{ }^{1}$ Lutfiah I. Al-Turk, $^{2}$ and Sharifah A. Al-Rajhi ${ }^{2}$ \\ ${ }^{1}$ Department of Statistics, College of Sciences, University of Jeddah, Jeddah 21551, Saudi Arabia \\ ${ }^{2}$ Department of Statistics, College of Sciences, King Abdulaziz University, Jeddah 21551, Saudi Arabia \\ Correspondence should be addressed to Norah N. Al-Mutairi; nnalmetere@uj.edu.sa
}

Received 13 July 2020; Revised 16 October 2020; Accepted 24 October 2020; Published 6 November 2020

Academic Editor: Emilio Gómez-Déniz

Copyright (c) 2020 Norah N. Al-Mutairi et al. This is an open access article distributed under the Creative Commons Attribution License, which permits unrestricted use, distribution, and reproduction in any medium, provided the original work is properly cited.

\begin{abstract}
Software reliability is an important feature that influences systems' reliability. Software reliability models are a common tool to evaluate software reliability quantitatively. Various reliability models have been suggested based on the NHPP (nonhomogeneous Poisson process). In this article, a new NHPP model based on the Lindley distribution is proposed. The mathematical formulas for its measures of reliability are obtained and graphically illustrated. The proposed model's parameters are estimated using both the NLSE (nonlinear least squares estimation) and the WNLSE (weighted nonlinear least squares estimation) methods. The model is then validated based on several different reliability datasets. The methods of estimation are evaluated and compared using three different criteria. The performance of the new model is also evaluated and compared, both objectively and subjectively, with three previously suggested models. The application results show that our new model demonstrates good performance in our selected failure data.
\end{abstract}

\section{Introduction}

The development of software systems is becoming more expensive and time-consuming because of their increasing complexity. Consequently, the reliable performance of software systems is becoming more important. Numerous SRGMs (software reliability growth models) with various assumptions have been proposed since the 1970s [1-3]. Several researchers have used reliability models based on the NHPP during the past years [4-8].

Kapur et al. [9] proposed a new SRGM based on Itô type of stochastic differential equation; the proposed model performs comparatively better than the existing NHPP models. Xu and Yao [10] also suggested a novel NHPP model based on the partial differential equation, and their suggested model exhibits a closer fitting to observation. $\mathrm{Li}$ and Yi [11] proposed a modified SRGM to reconsider the reliability of open source software and showed that it well fits the failure data and provides powerful prediction capability. Ramasamy and Lakshmanan [12] proposed the SRGM with infinite testing effort function. Recently, Al-Turk [13] proposed a NHPP model based on the two-parameter log-logistic distribution. The essential model characteristics were obtained, and the parameters of the model were estimated using the MLE (maximum likelihood estimation) and the NLSE methods. The results of the application indicate that the considered model gives a reasonable prediction capability for real studied datasets. Hui and Liu [14] proposed a SRGM based on Gaussian new distribution. The proposed model was confirmed by experiments to have a better fit and prediction performance than other reliability models.

In this article, we propose a new model that belongs to the NHPP class and based on the Lindley distribution. Several properties of the proposed model are outlined in Section 2 with graphical representations. These properties include MVF (mean value function), failure intensity, number of remaining faults, error detection rate, MTBF (mean time between failures), and conditional reliability. The NLSE and WNLSE methods are used for the purpose of estimating the proposed model parameters in Section 3. 
Application on real datasets is provided in Section 4. The last section concludes the article.

\section{NHPP Lindley Model}

2.1. Model Construction. A one-parameter Lindley distribution was suggested by Lindley [15] for the analysis of failure data. This model can capture failure data with different shapes of hazard rates. It has been studied by several authors as a good alternative to the exponential and Weibull distributions [16-18]. As with all statistical distributions, the Lindley distribution is specified by its PDF (probability density function),

$$
f(t)=\frac{\theta^{2}}{\theta+1}(1+t) e^{-\theta t}
$$

or its CDF (cumulative distribution function),

$$
F(t)=1-\frac{\theta+1+\theta t}{\theta+1} e^{-\theta t}
$$

where $t>0, \theta>0$, and $\theta$ is the shape parameter. The main aim of the NHPP model is to assess and predict the expected number of detected faults up to a specific point of time, which can be achieved using its MVF. Suppose $m(t)$ denotes the cumulative number of faults discovered at time $t$, and $F(t)$ is the distribution function of time between two successive failures, then the MVF of the NHPP model can be expressed as follows [5]:

$$
m(t)=a F(t)
$$

while the corresponding intensity function is given by

$$
\lambda(t)=\frac{\mathrm{d} m(t)}{\mathrm{d} t}=a f(t)
$$

where $a>0$ is the expected number of faults. By substituting equations (1) and (2) into equations (3) and (4), respectively, we get the MVF of the NHPP L (Lindley) model as follows:

$$
m(t)=a\left[\frac{(\theta+1)-(\theta+1+\theta t) e^{-\theta t}}{\theta+1}\right],
$$

and its corresponding intensity function as follows:

$$
\lambda(t)=\frac{a \theta^{2}}{\theta+1}(1+t) e^{-\theta t} .
$$

2.2. Model Characteristics. The NHPP model has very useful reliability measures for describing failure phenomena. In this section, the mathematical formulas of some of these measures for the new model will be given. First, the number of remaining faults of the NHPP L model is given by

$$
\begin{aligned}
n(t) & =a-m(t) \\
& =\frac{a(\theta+1+\theta t)}{\theta+1} e^{-\theta t},
\end{aligned}
$$

and then the error detection rate can be defined as follows:

$$
\begin{aligned}
d(t) & =\frac{\lambda(t)}{a-m(t)} \\
& =\frac{\theta^{2}(1+t)}{\theta+1+\theta t},
\end{aligned}
$$

while the MTBF is as follows:

$$
\begin{aligned}
\operatorname{MTBF} & =\frac{1}{\lambda(t)} \\
& =\frac{\theta+1}{a \theta^{2}(1+t) e^{-\theta t}} .
\end{aligned}
$$

The conditional reliability $R(x \mid t)$ is expressed by the probability that nondetected fault is found in the interval $(t, t+x)$, given that a fault occurred at time $t \geq 0 . x>0$ is the interval of operation time according to some practical or administrative requirements [19]. Mathematically, the conditional reliability of the NHPP L model can be obtained as follows:

$$
\begin{aligned}
R(x \mid t) & =\exp [-(m(t+x)-m(t))] \\
& =\exp \left[-\frac{a}{\theta+1}\left(\left(1-e^{-\theta x}\right)\left(e^{-\theta t}(\theta+1+\theta t)\right)-\theta x e^{-\theta(t+x)}\right)\right] .
\end{aligned}
$$

2.3. Graphs of the Model Characteristics. The plots of the NHPP L model's characteristics for different selected values of parameters are shown in Figures 1-6. Figure 1 illustrates the MVF which represents the variation of the number of faults detected with respect to time. From this figure, we can see that, initially, the faults detected during testing are very high but later on become stable, and also larger values of the parameter $a$ give higher MVF form. Figure 2 displays that the intensity function varies in shape over the different selected shape parameters, and it reaches a larger peak level with the larger value of the parameter $a$. The number of remaining errors function in Figure 3 decreases as the testing time increases; smaller values of the parameter $a$ give a lower form of the number of remaining errors function. In Figure 4 , the error detection rate function increases as the testing time increases; a larger value of the shape parameter gives a larger form of the failure occurrence rate per fault of the software function. The MTBF function in Figure 5 increases with the progress of the testing time. In Figure 6, we can see that as $t$ tends to infinity the conditional reliability becomes approximately 1 .

\section{Estimation of Model Parameters}

In this section, the NLSE and WNLSE methods are applied for the estimation of parameters of our proposed model.

3.1. The NLSE and WNLSE Methods. Assume that a software system is tested for $T$ units of time and $n$ faults were detected. Let $0<t_{1}<t_{2}<\cdots<t_{n}<T$ be the times at which the failures were observed. $m\left(t_{i} ; \Theta\right)$ is the MVF; and $\Theta$ is its unknown 


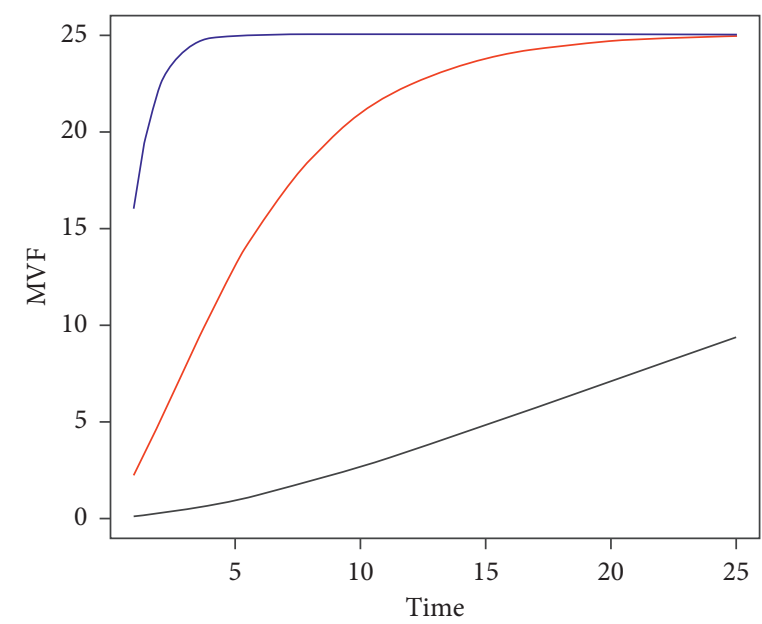

$$
\begin{aligned}
-a & =25, \theta=0.05 \\
-a & =25, \theta=0.3 \\
a & =25, \theta=1.5
\end{aligned}
$$

(a)

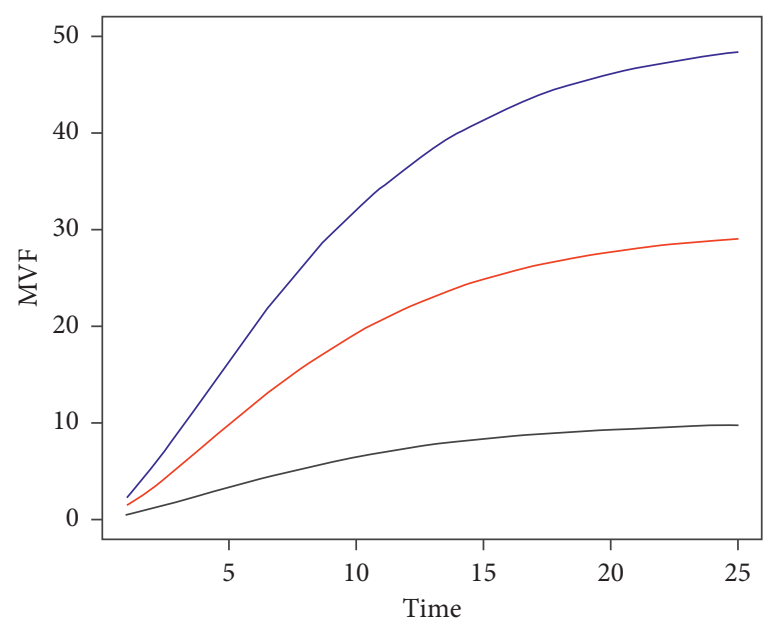

$$
\begin{aligned}
a & =10, \theta=0.2 \\
-a & =30, \theta=0.2 \\
-a & =50, \theta=0.2
\end{aligned}
$$

(b)

FIgURE 1: Plots of the MVF of the NHPP L model for (a) selected values of $\theta$ and (b) selected values of $a$.

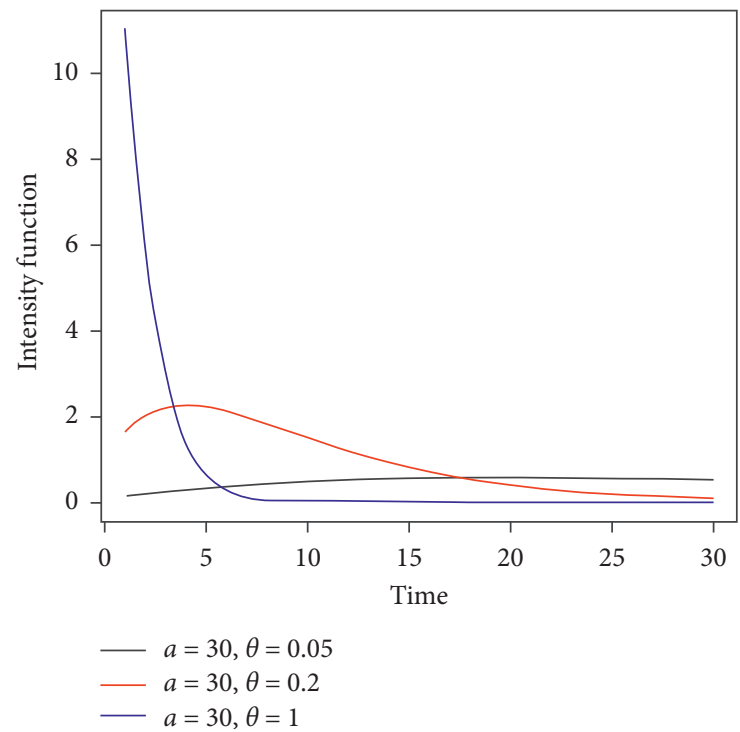

(a)

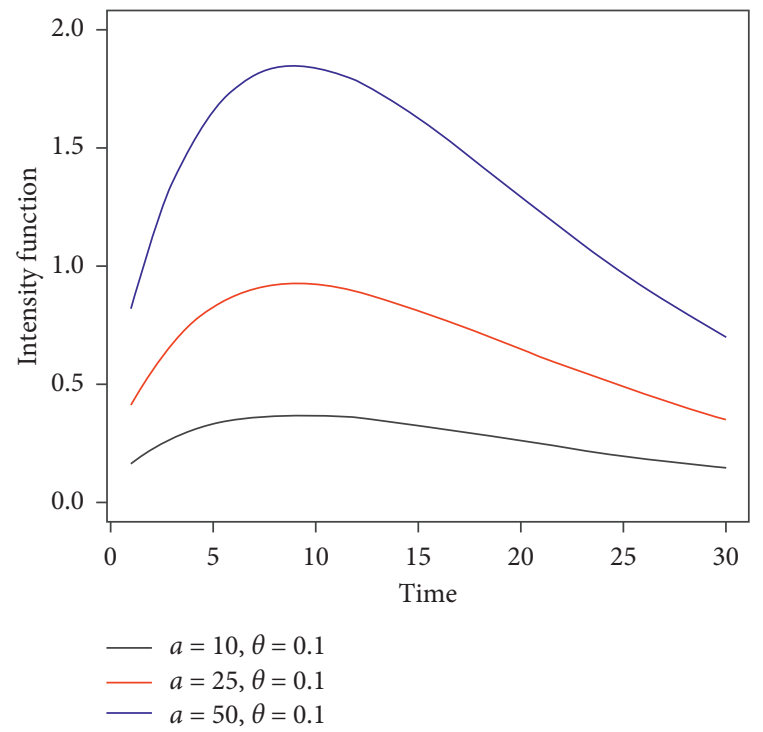

(b)

FIgURE 2: Plots of the intensity function of the NHPP L model for (a) selected values of $\theta$ and (b) selected values of $a$.

parameters. The parameters $\Theta$ are thus derived from $n$ observed data pairs: $\left(m_{0}, t_{0}\right),\left(m_{1}, t_{1}\right), \ldots,\left(m_{n}, t_{n}\right)$ where $m_{i}$ is the total number of faults detected within time $\left(0, t_{i}\right)$. Then, the NLSE method aims to minimize the following function:

$$
\operatorname{LSS}(\Theta)=\sum_{i=1}^{n} e_{i}^{2}=\sum_{i=1}^{n}\left(m_{i}-m\left(t_{i} ; \Theta\right)\right)^{2},
$$

while the WNLSE method aims to minimize the following function:

$$
\operatorname{LSS}_{W}(\Theta)=\sum_{i=1}^{n} w_{i}\left(m_{i}-m\left(t_{i} ; \Theta\right)\right)^{2},
$$

where $w_{i}>0$ and $(i=1, \ldots, n)$ are positive weights; $\sum_{i=1}^{n} w_{i}=$ $n[20]$.

3.2. The NLSE and WNLSE Methods for the NHPP L Model. For the NLSE, we substitute equation (5) in equation (11) as follows: 


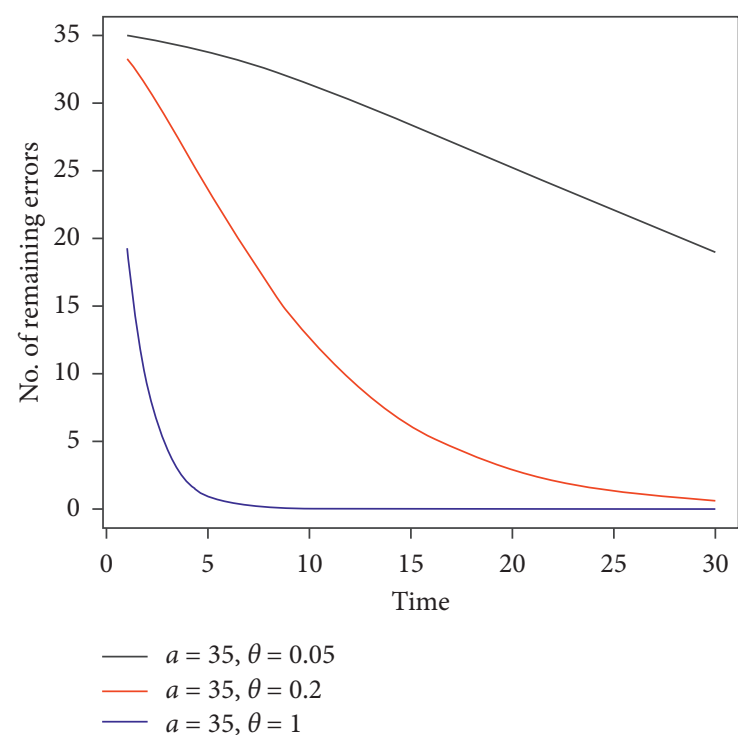

(a)

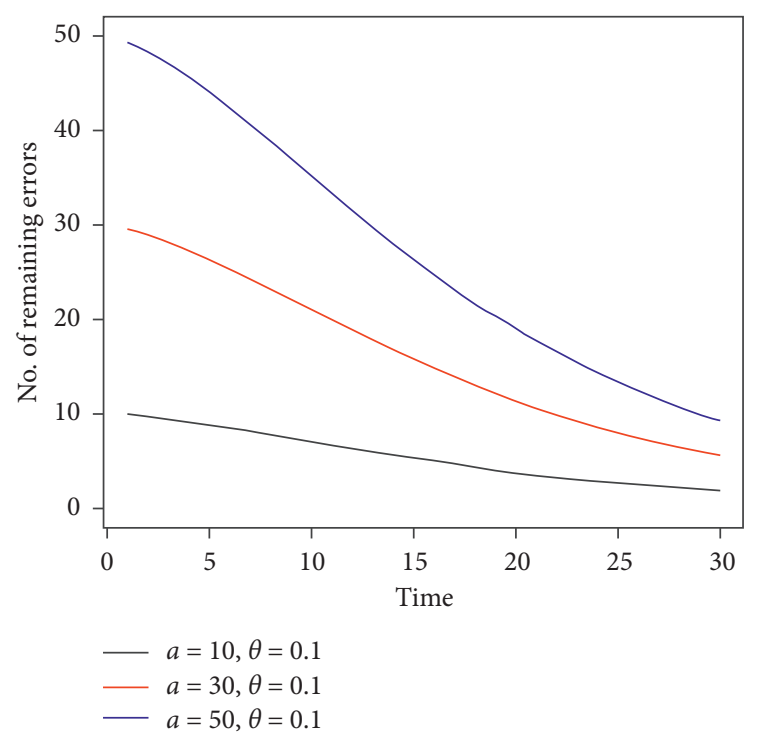

(b)

Figure 3: Plots of the number of remaining faults function of the NHPP L model for (a) selected values of $\theta$ and (b) selected values of $a$.

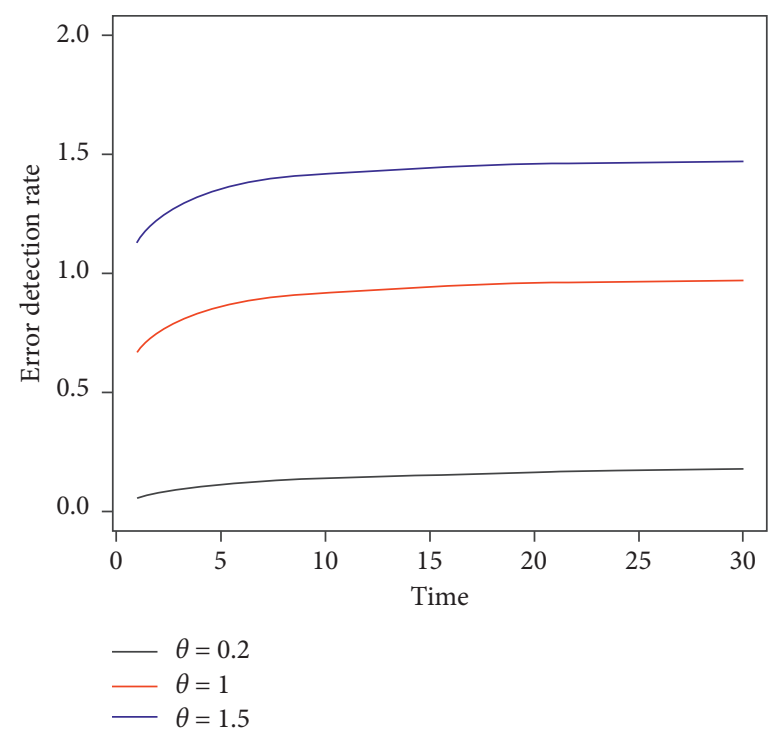

Figure 4: Plots of the error detection rate function of the NHPP L model for selected values of $\theta$.

$\operatorname{LSS}(a, \theta)=\sum_{i=1}^{n}\left(m_{i}-a\left[1-\frac{\left(\theta+1+\theta t_{i}\right)}{\theta+1} e^{-\theta t_{i}}\right]\right)^{2}$.

Taking the partial derivative of equation (13) with respect to $a$ and $\theta$, respectively, we get

$$
\begin{aligned}
& \frac{\mathrm{dLSS}(a, \theta)}{\mathrm{d} a}=-2 \sum_{i=1}^{n}\left(m_{i}-a\left[1-\frac{\left(\theta+1+\theta t_{i}\right)}{\theta+1} \cdot e^{-\theta t_{i}}\right]\right) \cdot\left(1-\frac{\left(\theta+1+\theta t_{i}\right)}{\theta+1} \cdot e^{-\theta t_{i}}\right) \\
& \frac{\mathrm{dLSS}(a, \theta)}{\mathrm{d} \theta}=2 \sum_{i=1}^{n}\left(\left(m_{i}-a\left[1-\frac{\left(\theta+1+\theta t_{i}\right)}{\theta+1} e^{-\theta t_{i}}\right]\right)\left[a\left(\frac{\left(\theta+1+\theta t_{i}\right)}{\theta+1}\left(-t e^{-\theta t_{i}}\right)+\frac{(\theta+1)\left(1+t_{i}\right)-\left(\theta+1+\theta t_{i}\right)}{(\theta+1)^{2}} e^{-\theta t_{i}}\right)\right]\right) .
\end{aligned}
$$




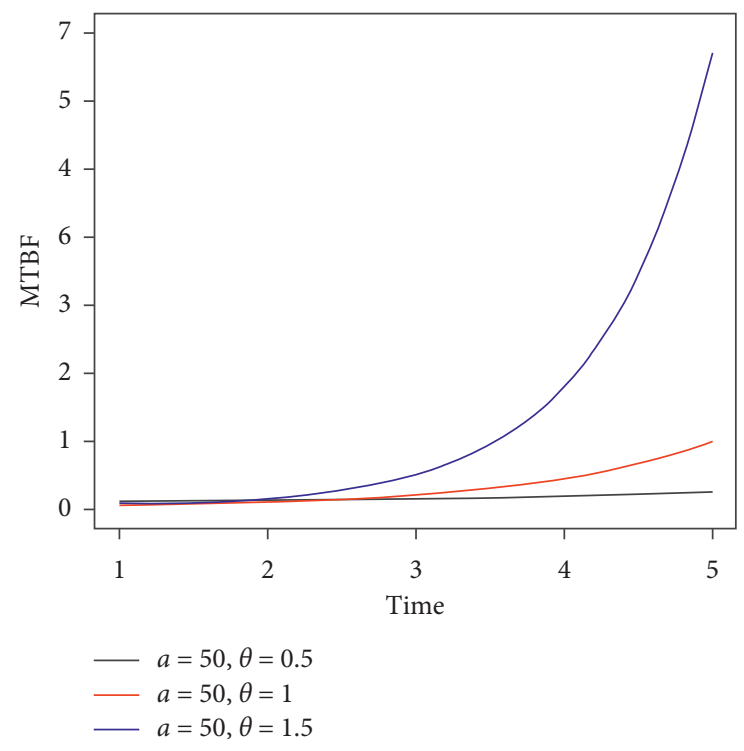

(a)

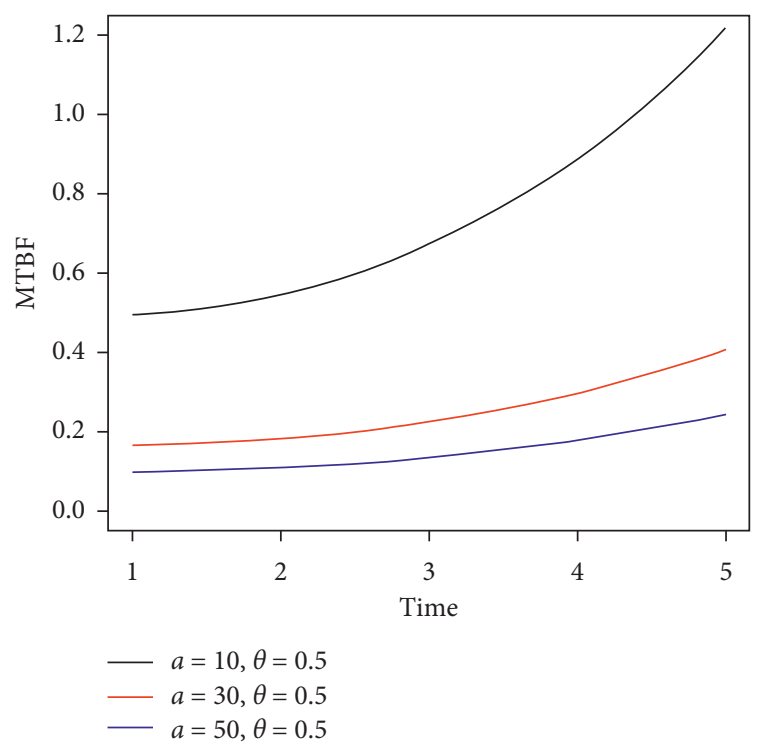

(b)

Figure 5: Plots of the MTBF function of the NHPP L model for (a) selected values of $\theta$ and (b) selected values of $a$.

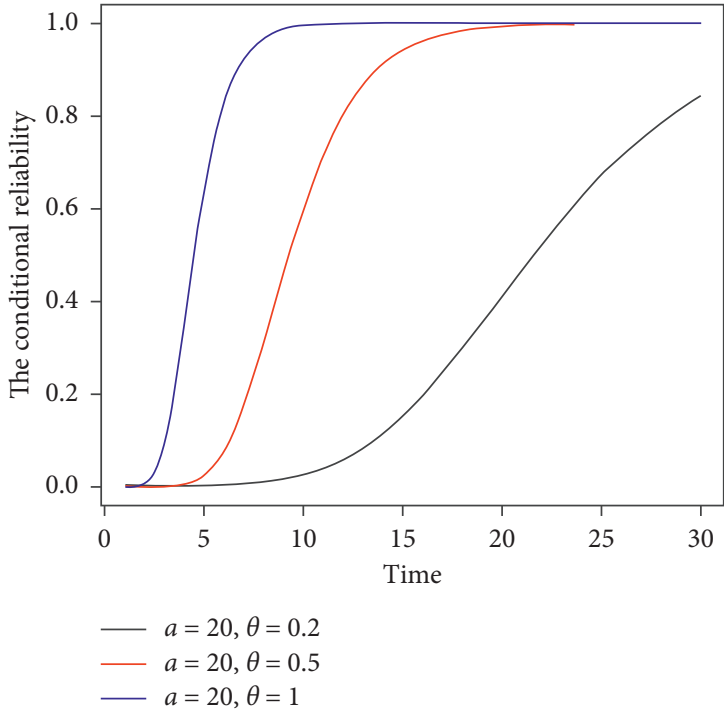

(a)

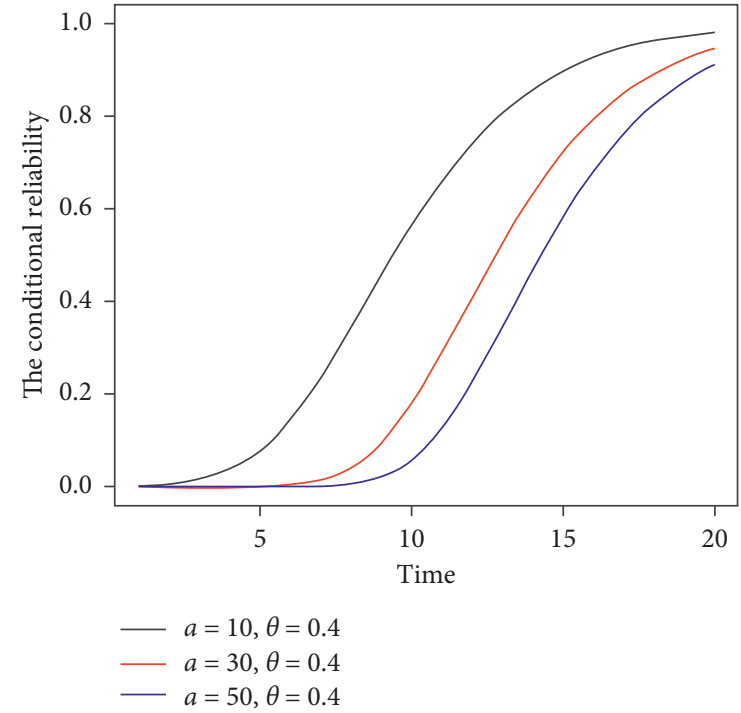

(b)

FIgUre 6: Plots of the conditional reliability of the NHPP L model for (a) selected values of $\theta$ and (b) selected values of $a$.

By setting the derivatives equal to zero, we get the following nonlinear equations:

$$
\begin{gathered}
\widehat{a}=\frac{\sum_{i=1}^{n} m_{i}\left(1-\left(\left(\theta+1+\theta t_{i}\right) /(\theta+1)\right) \cdot e^{-\theta t_{i}}\right)}{\sum_{i=1}^{n}\left(1-\left(\left(\theta+1+\theta t_{i}\right) /(\theta+1)\right) \cdot e^{-\theta t_{i}}\right)^{2},} \\
a \sum_{i=1}^{n} m_{i} \cdot t_{i} e^{-\theta t_{i}} \cdot\left(\frac{1}{(\theta+1)^{2}}-\frac{\left(\theta+1+\theta t_{i}\right)}{\theta+1}\right)+a^{2} \sum_{i=1}^{n}\left[1-\frac{\left(\theta+1+\theta t_{i}\right)}{\theta+1} e^{-\theta t_{i}}\right] \cdot t_{i} e^{-\theta t_{i}} \cdot\left(\frac{\left(\theta+1+\theta t_{i}\right)}{\theta+1}-\frac{1}{(\theta+1)^{2}}\right)=0 .
\end{gathered}
$$


The closed form expression for the NLS estimates of $\theta$ cannot be obtained. Consequently, an estimate of parameter $\theta$ can be obtained by numerically solving the nonlinear equation (16), and then by substituting this estimate in equation (15), the estimate of the parameter $a$ can be obtained.

For the WNLSE, we substitute equation (5) in equation (12), and thus we obtain

$$
\operatorname{LSS}_{W}(a, \theta)=\sum_{i=1}^{n} w_{i}\left(m_{i}-a\left[1-\frac{\left(\theta+1+\theta t_{i}\right)}{\theta+1} e^{-\theta t_{i}}\right]\right)^{2} .
$$

Taking the partial derivative of equation (17) with respect to $a$ and $\theta$, respectively, we get

$$
\begin{aligned}
& \frac{\mathrm{dLSS}_{W}(a, \theta)}{\mathrm{d} a}=-2 \sum_{i=1}^{n} w_{i}\left(m_{i}-\widehat{a}\left[1-\frac{\left(\theta+1+\theta t_{i}\right)}{\theta+1} \cdot e^{-\theta t_{i}}\right]\right) \cdot\left(1-\frac{\left(\theta+1+\theta t_{i}\right)}{\theta+1} \cdot e^{-\theta t_{i}}\right) \\
& \frac{\mathrm{dLSS}_{W}(a, \theta)}{\mathrm{d} \theta}=2 \sum_{i=1}^{n} w_{i}\left(\left(m_{i}-a\left[1-\frac{\left(\theta+1+\theta t_{i}\right)}{\theta+1} e^{-\theta t_{i}}\right]\right)\left[a\left(\frac{\left(\theta+1+\theta t_{i}\right)}{\theta+1}\left(-t e^{-\theta t_{i}}\right)+\frac{(\theta+1)\left(1+t_{i}\right)-\left(\theta+1+\theta t_{i}\right)}{(\theta+1)^{2}} e^{-\theta t_{i}}\right)\right]\right) .
\end{aligned}
$$

By setting the derivatives equal to zero, we have the following nonlinear equations:

$$
\begin{gathered}
\widehat{a}=\frac{\sum_{i=1}^{n} w_{i} m_{i}\left(1-\left(\left(\theta+1+\theta t_{i}\right) /(\theta+1)\right) \cdot e^{-\theta t_{i}}\right)}{\sum_{i=1}^{n} w_{i}\left(1-\left(\left(\theta+1+\theta t_{i}\right) /(\theta+1)\right) \cdot e^{-\theta t_{i}}\right)^{2},} \\
a \sum_{i=1}^{n} w_{i} m_{i} t_{i} e^{-\theta t_{i}} \cdot\left(\frac{1}{(\theta+1)^{2}}-\frac{\left(\theta+1+\theta t_{i}\right)}{\theta+1}\right)+a^{2} \sum_{i=1}^{n} w_{i}\left[1-\frac{\left(\theta+1+\theta t_{i}\right)}{\theta+1} e^{-\theta t_{i}}\right] \cdot t_{i} e^{-\theta t_{i}} \cdot\left(\frac{\left(\theta+1+\theta t_{i}\right)}{\theta+1}-\frac{1}{(\theta+1)^{2}}\right)=0 .
\end{gathered}
$$

Closed form expression for the WNLS estimate of $\theta$ cannot be obtained. By solving equation (20) using the Gauss-Newton method, we obtain the value of the estimate, and then by substituting this estimate in equation (19), the estimate of the parameter $a$ can be obtained.

\section{Application to Failure Data}

In this section, examples of real data are used to compare the two considered methods of estimation for the proposed model. Also, we perform a comparative study to evaluate the effectiveness of the proposed model with three of the previously existing models. Useful results based on the studied real datasets are presented and discussed at the end of this section. To facilitate mathematical computation, a software tool was developed using $\mathrm{R}$ language version 3.6.1.

4.1. Datasets. Nine published datasets with different sizes were chosen for our evaluation study. References for the selected datasets are shown in Table 1.

4.2. Models. In addition to our proposed model (NHPP L), three other well-known reliability models are considered, and the names and MVFs of these models are listed in Table 2 .
4.3. Evaluation Criteria. To check the performance of the considered models, we used the following three criteria based on equations (21)-(23). The mean square error (MSE) is the variation between the predicted values and the actual observations. It is defined as [19]

$$
\operatorname{MSE}=\sum_{i=1}^{n} \frac{\left(\widehat{m}\left(t_{i}\right) t-n m_{i}\right)^{2}}{n-k}
$$

where $\widehat{m}\left(t_{i}\right)$ is the estimated number of faults at time $t_{i}$ obtained from the considered model; $m_{i}$ is the total number of faults detected within time $\left(0, t_{i}\right),(i=1, \ldots, n) ; n$ is the number of observations; and $k$ is the number of parameters. A lower value of the MSE indicates more confidence in the model and thus better performance. The variance is defined as follows [29, 30]:

$$
\text { variance }=\sqrt{\frac{1}{n-1} \sum_{i=1}^{n}\left(m_{i}-\widehat{m}\left(t_{i}\right)-\text { Bais }\right)^{2}},
$$

where the bias is defined as $\left|\sum_{i=1}^{n}\left(m\left(t_{i}\right)-m_{i}\right) / n\right|$. The average of the prediction faults is referred to as the prediction bias, and its standard deviation is often used as a measure of the variance in the predictions. The small value of variance indicates that the model fits the data well. The coefficient of determination $\left(R^{2}\right)$ can measure how precise the fit is in describing the deviation of the data. It is defined as [19] 
TABLE 1: References for the datasets.

\begin{tabular}{lcc}
\hline Dataset & References & Number of faults \\
\hline 1 & Hayakawa and Telfar [21] & 30 \\
2 & Li and Pham [22] & 14 \\
3 & Li and Pham [22] & 60 \\
4 & Xie et al. [23] & 30 \\
5 & Wang et al. [24] & 21 \\
6 & Hurtado et al. [25] & 23 \\
7 & Hurtado et al. [25] & 15 \\
8 & Hurtado et al. [25] & 14 \\
9 & Liu et al. [26] & 31 \\
\hline
\end{tabular}

TABLE 2: The model names and MVFs.

\begin{tabular}{lc}
\hline Model name & MVF \\
\hline $\begin{array}{l}\text { Goel-Okumoto } \\
(\text { GO }) \text { model }\end{array}$ & $m(t)=a\left(1-e^{-b t}\right)$ \\
$\begin{array}{l}4,27] \\
\text { Delayed S-shaped } \\
\text { model [5, 28] }\end{array}$ & $m(t)=a\left(1-(1+b t) e^{-b t}\right)$ \\
$\begin{array}{l}\text { Inflection } \\
\text { S-shaped model }\end{array}$ & $m(t)=\left(\left(a\left(1-e^{-b t}\right)\right) /\left(1+\beta e^{-b t}\right)\right)$ \\
[6] & \\
NHPP L model & $m(t)=a\left[\left((\theta+1)-(\theta+1+\theta t) e^{-\theta t}\right) /(\theta+1)\right]$ \\
\hline
\end{tabular}

where $\beta>0$ is the inflection factor; $a>0$ is the expected number of software faults to be eventually detected; $b$ is a constant of proportionality; and $\theta$ is the shape parameter for the Lindley distribution.

$$
R^{2}=1-\frac{\sum_{i=1}^{n}\left(m_{i}-\widehat{m}\left(t_{i}\right)\right)^{2}}{\sum_{i=1}^{n}\left(m_{i}-\sum_{j=1}^{n}\left(m_{j} / n\right)\right)^{2}} .
$$

Values for this coefficient range from 0 to 1 . The value of $R^{2}$ closest to 1 indicates the best model.

\subsection{Results and Discussion}

4.4.1. Comparative Study of the Estimation Methods. This section evaluates the performance of the NLSE and WNLSE methods for the NHPP L model based on eight datasets. The results are shown in Table 3. From the evaluation criteria values in Table 3, we derived the following conclusions:

(i) The NHPP L model provides values indicative of a better model for most of the evaluation criteria in most cases when using the WNLSE method.

(ii) The different evaluation criteria gave different results, and this indicates the necessity to study several criteria during the comparison.

Figures 7 and 8 illustrate the actual and fitted curves of software failures using the NLSE and WNLSE methods. According to these figures, we can see our new model provides a good fit for all considered datasets when using either the NLSE or WNLSE methods. In particular, the proposed model is more suitable for modeling the failure datasets when using the WNLSE method rather than the NLSE method.
TABLE 3: Estimated values of parameters and comparison criteria results of the NHPP L model.

\begin{tabular}{lcccccc}
\hline \multirow{2}{*}{ Dataset } & \multirow{2}{*}{$\begin{array}{c}\text { Method of } \\
\text { estimation }\end{array}$} & \multicolumn{2}{c}{ Estimated } & \multicolumn{2}{c}{ Evaluation criteria } \\
& & $\hat{a}$ & $\hat{\theta}$ & MSE & Variance & $R^{2}$ \\
\hline \multirow{2}{*}{ DS1 } & NLSE & 33.230 & 0.160 & 2.264 & 2.418 & 0.989 \\
& WNLSE & 28.085 & 0.205 & 1.632 & 3.887 & 0.978 \\
\hline \multirow{2}{*}{ DS2 } & NLSE & 88.940 & 0.018 & 0.271 & 0.525 & 0.985 \\
& WNLSE & 55.630 & 0.023 & 0.774 & 0.603 & 0.952 \\
\hline \multirow{2}{*}{ DS3 } & NLSE & 67.500 & 0.026 & 13.077 & 13.985 & 0.956 \\
& WNLSE & 61.170 & 0.031 & 3.543 & 16.947 & 0.988 \\
\hline \multirow{2}{*}{ DS4 } & NLSE & 27.180 & 0.012 & 1.392 & 1.724 & 0.982 \\
& WNLSE & 27.240 & 0.012 & 0.679 & 1.739 & 0.991 \\
\hline \multirow{2}{*}{ DS5 } & NLSE & 29.490 & 0.014 & 0.747 & 0.924 & 0.983 \\
& WNLSE & 27.090 & 0.015 & 0.486 & 0.944 & 0.988 \\
\hline \multirow{2}{*}{ DS6 } & NLSE & 64.490 & 0.001 & 4.162 & 4.279 & 0.924 \\
& WNLSE & 30.590 & 0.001 & 2.689 & 5.187 & 0.942 \\
\hline \multirow{2}{*}{ DS7 } & NLSE & 17.540 & 0.002 & 1.903 & 1.744 & 0.926 \\
& WNLSE & 15.017 & 0.002 & 1.134 & 2.446 & 0.955 \\
\hline \multirow{2}{*}{ DS8 } & NLSE & 11.550 & 0.003 & 2.196 & 2.196 & 0.909 \\
& WNLSE & 11.556 & 0.004 & 0.525 & 2.329 & 0.977 \\
\hline
\end{tabular}

4.4.2. Comparing the Performance of Various SRGMs for Some Real Datasets. Since the proposed model is new concerning the predication/estimation of software reliability, we compared its accuracy with some well-known and widely used SRGMs, namely, the GO model, delayed S-shaped model, and inflection S-shaped model. Our comparative study is based on five datasets, DS2, DS3, DS4, DS5, and DS9, and used the WNLSE as the method of estimation. The Kolmogorov-Smirnov test was used to check and compare the fit between these datasets and our studied reliability models. The results are presented in Table 4 . From the table, we can observe the following:

(i) The MSE values for all studied models are very close, indicating that all studied models have the ability to describe the five selected systems effectively with minor differences between them in terms of their performance. The NHPP L model ranked the second for DS2, DS3, and DS5 while it ranked the first for DS4 and the third for DS9.

(ii) The values for the coefficient of determination $\left(R^{2}\right)$ for all studied models are close to 1 . Therefore, it can 


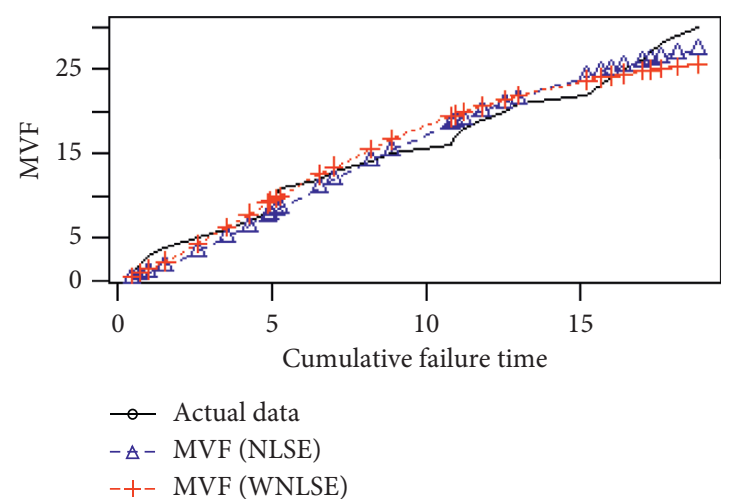

(a)

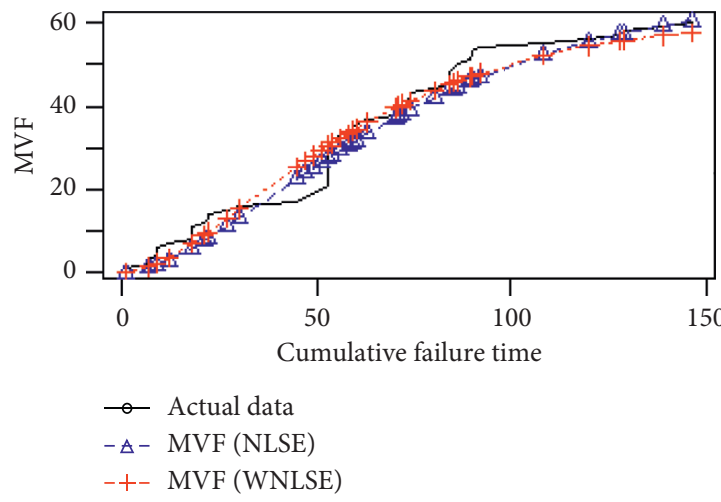

(c)

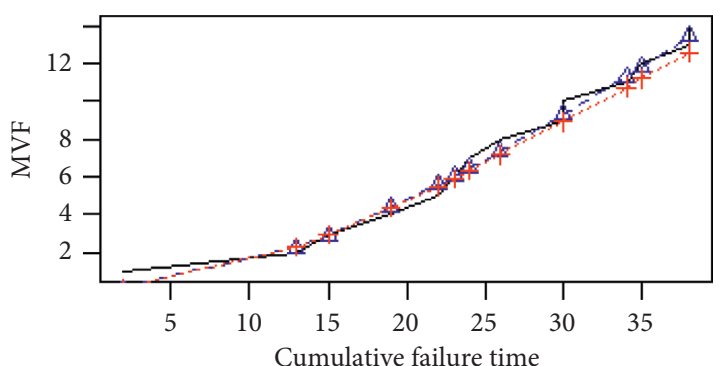

$\rightarrow$ Actual data

- - MVF (NLSE)

-+- MVF (WNLSE)

(b)

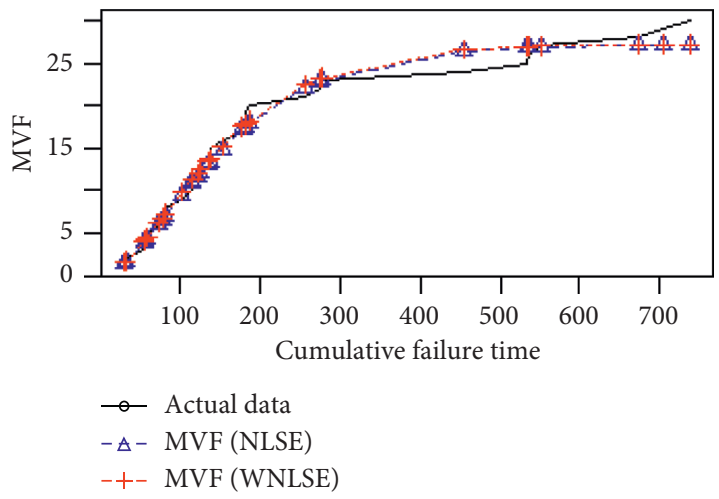

(d)

Figure 7: Comparison between methods of estimation for the NHPP L model for (a) DS1, (b) DS2, (c) DS3, and (d) DS4.
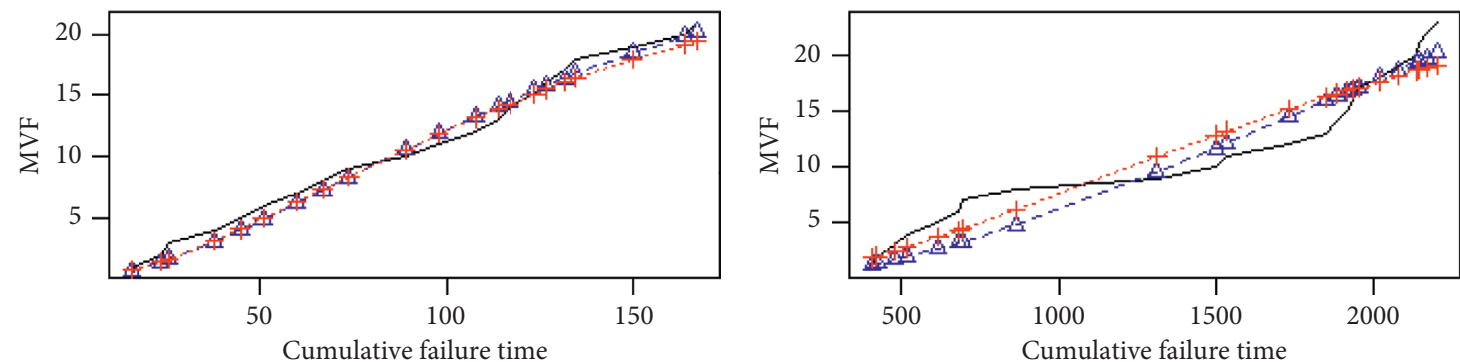

$$
\begin{aligned}
& \rightarrow \text { Actual data } \\
& - \text { - }- \text { MVF (NLSE) } \\
& -+- \text { MVF (WNLSE) }
\end{aligned}
$$

(a)

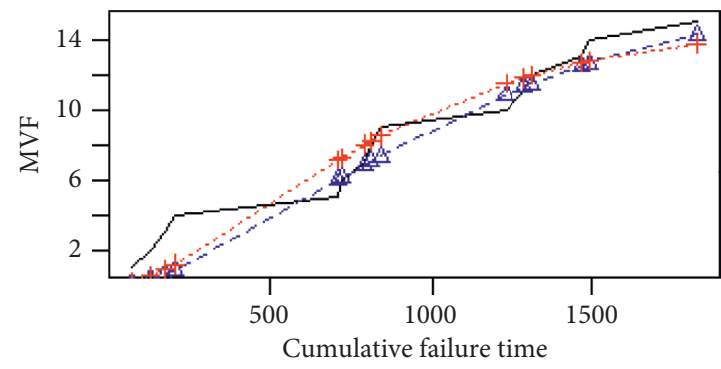

$$
\begin{aligned}
& \text { - Actual data } \\
& -A-\text { MVF (NLSE) } \\
& -+- \text { MVF (WNLSE) }
\end{aligned}
$$

(c)

$$
\begin{aligned}
& \rightarrow \text { Actual data } \\
& - \text { - }- \text { MVF (NLSE) } \\
& -+- \text { MVF (WNLSE) }
\end{aligned}
$$

(b)

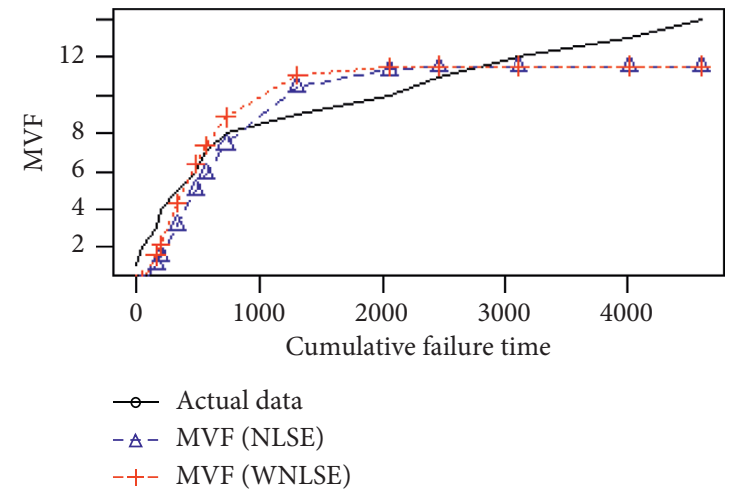

(d)

FIgURE 8: Comparison between methods of estimation for the NHPP L model for (a) DS5, (b) DS6, (c) DS7, and (d) DS8. 
TABLE 4: Comparison results of different models for different datasets.

\begin{tabular}{|c|c|c|c|c|c|}
\hline Criteria & Dataset & Goel-Okumoto (GO) & Delayed S-shaped & Inflection S-shaped & Proposed model (NHPP L) \\
\hline \multirow{5}{*}{ MSE } & DS2 & 1.316 & 0.909 & 0.458 & 0.774 \\
\hline & DS3 & 6.505 & 2.303 & 4.491 & 3.543 \\
\hline & DS4 & 3.686 & 0.689 & 1.055 & 0.679 \\
\hline & DS5 & 0.545 & 0.546 & 0.291 & 0.486 \\
\hline & DS9 & 4.216 & 0.753 & 0.658 & 0.774 \\
\hline \multirow{5}{*}{ Variance } & DS2 & 2.635 & 0.667 & 0.608 & 0.603 \\
\hline & DS3 & 17.760 & 15.910 & 12.310 & 16.940 \\
\hline & DS4 & 4.099 & 2.355 & 2.442 & 1.739 \\
\hline & DS5 & 1.065 & 1.483 & 0.685 & 0.944 \\
\hline & DS9 & 5.296 & 2.327 & 2.473 & 2.429 \\
\hline \multirow{5}{*}{$R^{2}$} & DS2 & 0.879 & 0.949 & 0.977 & 0.952 \\
\hline & DS3 & 0.976 & 0.992 & 0.985 & 0.987 \\
\hline & DS4 & 0.949 & 0.991 & 0.986 & 0.991 \\
\hline & DS5 & 0.984 & 0.986 & 0.993 & 0.987 \\
\hline & DS9 & 0.945 & 0.991 & 0.992 & 0.991 \\
\hline
\end{tabular}

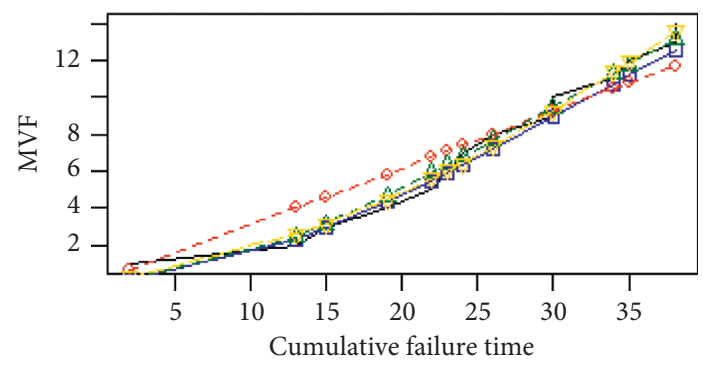

$\begin{array}{rrl}\rightarrow-\text { Actual data } & -\Delta- & \text { Delayed S-shaped } \\ & \text { model } \\ \rightarrow-\text { Proposed model } & \rightarrow- & \text { Inflection S-shaped } \\ -\bullet-\text { GO model } & & \text { model }\end{array}$

(a)

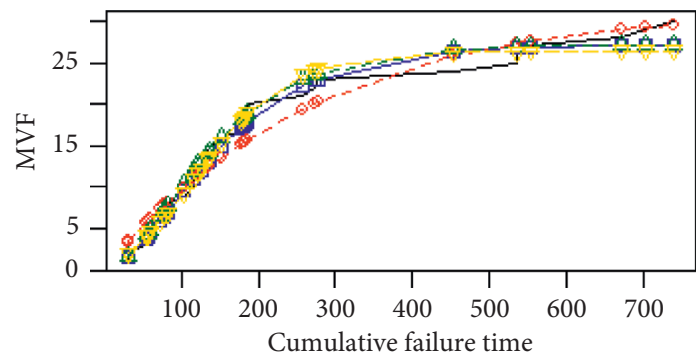

$\begin{array}{lrl}\rightarrow-\text { Actual data } & -\Delta- & \text { Delayed S-shaped } \\ & \text { model } \\ \rightarrow-\text { Proposed model } & & \text { Inflection S-shaped } \\ -\ominus-\text { GO model } & -\nabla-\begin{array}{l}\text { model }\end{array}\end{array}$

(c)

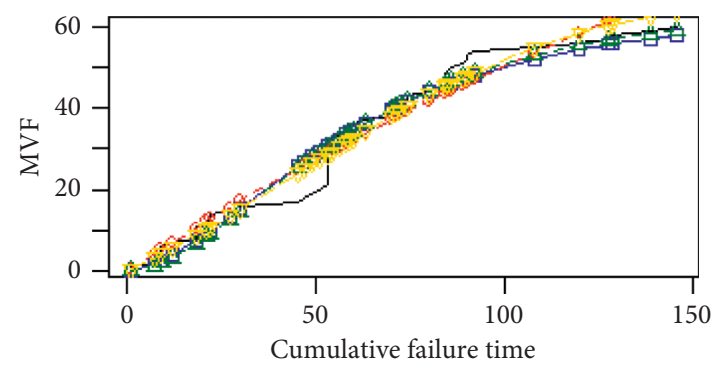

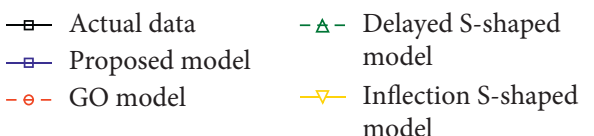

(b)

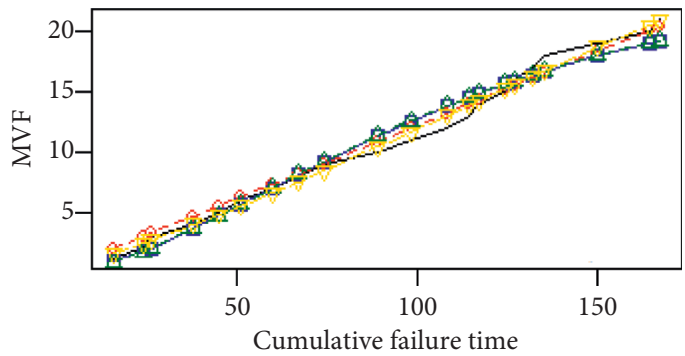

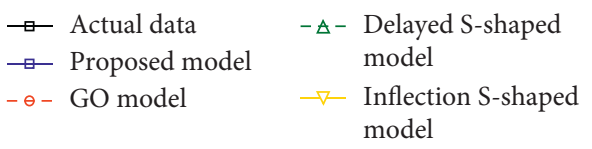

(d)

Figure 9: Continued. 


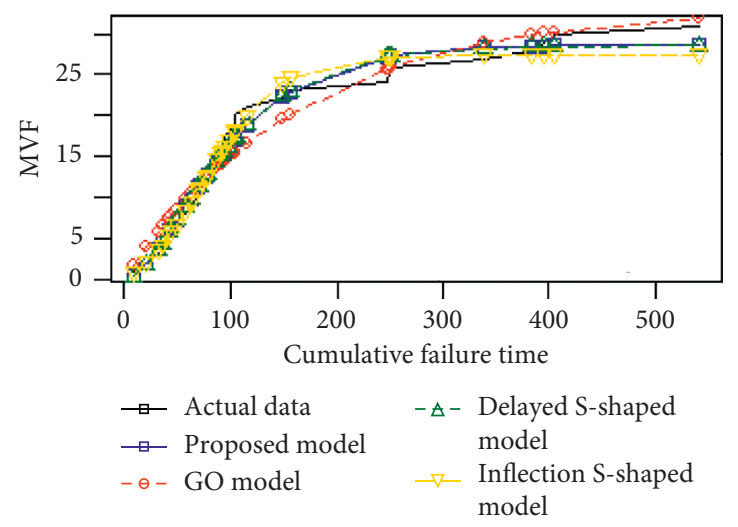

(e)

FIGURE 9: Actual and prediction results for (a) DS2, (b) DS3, (c) DS4, (d) DS5, and (e) DS9.

be said that all studied models are suitable for modeling the considered software projects.

Figure 9 illustrates the actual and prediction results based on the four considered models. According to these figures, we can see that all the selected models are well-fitted to the studied failure data. In particular, the proposed model is one of the most suitable for modeling the selected datasets.

\section{Conclusions}

In this article, we propose a new reliability model based on the Lindley distribution. Several essential characteristics of our proposed model, the NHPP L model, were obtained. The considered model parameters were estimated using the NLSE and WNLSE methods. The performance of the estimators for each studied method was evaluated using different criteria based on eight datasets. A comparative study between the proposed model and three other common models was conducted based on five real datasets. The WNLSE method was determined to have better performance than the NLSE method for the chosen failure datasets. Thus, it is recommended that the WNLSE method be used with the NHPP models. The performance of the NHPP L model is encouraging in comparison with other selected models. The present study can be extended by incorporating SRGMs with learning effects to increase the flexibility of models and to enhance their capability for accurately describing software failure phenomena.

\section{Abbreviations}

SRGMs: Software reliability growth models

NHPP: Nonhomogeneous Poisson process

PDF: Probability density function

CDF: Cumulative distribution function

MVF: Mean value function

MTBF: Mean time between failures

MSE: $\quad$ Mean of squared errors

$R^{2}$ : Coefficient of multiple determination

NLSE: Nonlinear least square estimation

WNLSE: Weighted nonlinear least square estimation

MLE: Maximum likelihood estimation.

\section{Data Availability}

Previously published data were used to support this study. These prior studies are cited at relevant places within the text as references.

\section{Conflicts of Interest}

The authors declare that they have no conflicts of interest regarding the publication of this paper.

\section{References}

[1] W. D. Jones, "Reliability models for very large software systems in industry," in Proceedings of the 1991 International Symposium on Software Reliability Engineering, IEEE, Austin, TX, USA, May 1991.

[2] M. Xie, Software Reliability Modelling, World Scientific, Singapore, 1991.

[3] H. Pham, Software Reliability, Springer Science \& Business Media, Berlin, Germany, 2000.

[4] A. L. Goel and K. Okumoto, "Time-dependent error-detection rate model for software reliability and other performance measures," IEEE Transactions on Reliability, vol. R-28, no. 3, pp. 206-211, 1979.

[5] S. Yamada, M. Ohba, and S. Osaki, "S-shaped reliability growth modeling for software error detection," IEEE Transactions on Reliability, vol. R-32, no. 5, pp. 475-484, 1983.

[6] M. Ohba, "Inflection S-shaped software reliability growth model," in Stochastic Models in Reliability Theory, pp. 144-162, Springer, Berlin, Germany, 1984.

[7] X. Zhang, X. Teng, and H. Pham, "Considering fault removal efficiency in software reliability assessment," IEEE Transactions on Systems, Man, and Cybernetics-Part A: Systems and Humans, vol. 33, no. 1, pp. 114-120, 2003.

[8] X. Teng and H. Pham, "A software cost model for quantifying the gain with considerations of random field environments," IEEE Transactions on Computers, vol. 53, no. 3, pp. 380-384, 2004.

[9] P. K. Kapur, S. Anand, S. Yamada, and V. S. S. Yadavalli, "Stochastic differential equation-based flexible software reliability growth model," Mathematical Problems in Engineering, vol. 2009, Article ID 581383, 15 pages, 2009.

[10] J. Xu and S. Yao, "Software reliability growth model with partial differential equation for various debugging processes," 
Mathematical Problems in Engineering, vol. 2016, Article ID 2476584, 13 pages, 2016.

[11] F. Li and Z.-L. Yi, "A new software reliability growth model: multigeneration faults and a power-law testing-effort function," Mathematical Problems in Engineering, vol. 2016, Article ID 9276093, 13 pages, 2016.

[12] S. Ramasamy and I. Lakshmanan, "Machine learning approach for software reliability growth modeling with infinite testing effort function," Mathematical Problems in Engineering, vol. 2017, Article ID 8040346, 6 pages, 2017.

[13] L. I. Al-Turk, "Characteristics and application of the NHPP log-logistic reliability model," International Journal of Statistics and Probability, vol. 8, no. 1, pp. 44-55, 2019.

[14] Z. Hui and X. Liu, "Research on software reliability growth model based on Gaussian new distribution," Procedia Computer Science, vol. 166, pp. 73-77, 2020.

[15] D. V. Lindley, "Fiducial distributions and Bayes' theorem," Journal of the Royal Statistical Society: Series B (Methodological), vol. 20, no. 1, pp. 102-107, 1958.

[16] M. E. Ghitany, F. Alqallaf, D. K. Al-Mutairi, and H. A. Husain, "A two-parameter weighted Lindley distribution and its applications to survival data," Mathematics and Computers in Simulation, vol. 81, no. 6, pp. 1190-1201, 2011.

[17] M. E. Ghitany, B. Atieh, and S. Nadarajah, "Lindley distribution and its application," Mathematics and Computers in Simulation, vol. 78, no. 4, pp. 493-506, 2008.

[18] J. Mazucheli and J. A. Achcar, "The Lindley distribution applied to competing risks lifetime data," Computer Methods and Programs in Biomedicine, vol. 104, no. 2, pp. 188-192, 2011.

[19] K.-C. Chiu, Y.-S. Huang, and T.-Z. Lee, "A study of software reliability growth from the perspective of learning effects," Reliability Engineering \& System Safety, vol. 93, no. 10, pp. 1410-1421, 2008.

[20] T. Ishii, T. Dohi, and H. Okamura, "Software reliability prediction based on least squares estimation," Quality Technology \& Quantitative Management, vol. 9, no. 3, pp. 243-264, 2012.

[21] Y. Hayakawa and G. Telfar, "Mixed Poisson-type processes with application in software reliability," Mathematical and Computer Modelling, vol. 31, no. 10-12, pp. 151-156, 2000.

[22] Q. Li and H. Pham, "NHPP software reliability model considering the uncertainty of operating environments with imperfect debugging and testing coverage," Applied Mathematical Modelling, vol. 51, pp. 68-85, 2017.

[23] M. Xie, T. N. Goh, and P. Ranjan, "Some effective control chart procedures for reliability monitoring," Reliability Engineering \& System Safety, vol. 77, no. 2, pp. 143-150, 2002.

[24] J. Wang, Z. Wu, Y. Shu, and Z. Zhang, "An optimized method for software reliability model based on nonhomogeneous Poisson process," Applied Mathematical Modelling, vol. 40, no. 13-14, pp. 6324-6339, 2016.

[25] J. L. Hurtado, F. Joglar, and M. Modarres, "Generalized renewal process: models, parameter estimation and applications to maintenance problems," International Journal of Performability Engineering, vol. 1, no. 1, pp. 37-50, 2005.

[26] J. Liu, Y. Liu, and M. Xu, "Parameter estimation of JelinskiMoranda model based on weighted nonlinear least squares and heteroscedasticity," 2015, https://arxiv.org/abs/1503. 00094.

[27] A. L. Goel, "Software error detection model with applications," Journal of Systems and Software, vol. 1, pp. 243-249, 1979.
[28] S. Yamada, M. Ohba, and S. Osaki, "S-shaped software reliability growth models and their applications," IEEE Transactions on Reliability, vol. R-33, no. 4, pp. 289-292, 1984.

[29] K. Pillai and V. S. Sukumaran Nair, "A model for software development effort and cost estimation," IEEE Transactions on Software Engineering, vol. 23, no. 8, pp. 485-497, 1997.

[30] C.-Y. Huang and S.-Y. Kuo, "Analysis of incorporating logistic testing-effort function into software reliability modeling," IEEE Transactions on Reliability, vol. 51, no. 3, pp. 261-270, 2002. 